TRANSACTIONS OF THE

AMERICAN MATHEMATICAL SOCIETY

Volume 364, Number 7, July 2012, Pages 3805-3827

S 0002-9947(2012)05563-2

Article electronically published on February 20, 2012

\title{
AN INTRINSIC APPROACH IN THE CURVED $n$-BODY PROBLEM. THE POSITIVE CURVATURE CASE
}

\author{
ERNESTO PÉREZ-CHAVELA AND J. GUADALUPE REYES-VICTORIA
}

\begin{abstract}
We consider the gravitational motion of $n$ point particles with masses $m_{1}, m_{2}, \ldots, m_{n}>0$ on surfaces of constant positive Gaussian curvature. Using stereographic projection, we express the equations of motion defined on the two-dimensional sphere of radius $R$ in terms of the intrinsic coordinates of the complex plane endowed with a conformal metric. This new approach allows us to derive the algebraic equations that characterize relative equilibria. The second part of the paper brings new results about necessary and sufficient conditions for the existence of relative equilibria in the cases $n=2$ and $n=3$.
\end{abstract}

\section{INTRODUCTION}

The equations of motion for the $n$-body problem in spaces of nonzero constant Gaussian curvature, also called the curved $n$-body problem, were first obtained by Florin Diacu, Ernesto Pérez-Chavela, and Manuele Santoprete [3. These authors used the cotangent potential and studied the Euler-Lagrange equations with the respective constraints that maintain the particles on the corresponding imbedded surfaces of constant curvature $\mathbf{K}$ (the 2 -sphere of radius $1 / \sqrt{\mathbf{K}}$ in $\mathbb{R}^{3}$ if $\mathbf{K}>0$, or the upper sheet of the hyperboloid $x^{2}+y^{2}-z^{2}=\mathbf{K}^{-1}$ in the Minkowski space $\mathbb{R}_{1}^{3}$ if $\mathbf{K}<0$ ). The paper $[3$ is the first work that analyzes the solutions of certain types of relative equilibria in spaces of constant curvature, that is, solutions for which the mutual distances among the particles are constant during the motion. Since any rotation in $\mathbb{R}^{3}$ is around a suitable fixed axis, and since the cotangent potential is invariant under the whole group of isometries $S O(3)$, the authors of [3] studied, without loss of generality, some solutions of relative equilibria which are invariant under the action of the subgroup

$$
A(t)=\left(\begin{array}{ccc}
\cos t & \sin t & 0 \\
-\sin t & \cos t & 0 \\
0 & 0 & 1
\end{array}\right),
$$

contained in the isometry group $S O(3)$ of the sphere for each $t \in \mathbb{R}$.

In the case $\mathbf{K}>0$, the authors of [3] proved that if three particles are initially at the vertices of an equilateral triangle, in the plane $z=$ constant, then the bodies generate a relative equilibrium only if the masses of the particles are equal. They also proved that if three particles are on the same rotating meridian, with one mass

Received by the editors November 26, 2010 and, in revised form, January 25, 2011.

2010 Mathematics Subject Classification. Primary 70F15, 34A26; Secondary 70F10, 70F07.

Key words and phrases. Differential geometry, Riemannian conformal metric.

Both authors thank the anonymous referees for their deep review of the original version and for their valuable comments and suggestions that helped us to improve this work. This work has been partially supported by CONACYT, México, Grant 128790.

(C)2012 American Mathematical Society 3805

Reverts to public domain 28 years from publication 
$M$ between the other two particles with equal mass $m$ symmetrically located on the meridian, then this configuration generates a relative equilibrium. In the above results the authors obtained those periodic orbits by fixing the initial configuration.

Following the ideas of [3], our first approach was to study the solutions $q(t)$ that are invariant to (1.1), that is, solutions of the equations of motion of the form $Q(t)=A(t) q(t)$. We thus found conditions to obtain relative equilibria, but unfortunately the equation in terms of $A(t), \dot{A}(t), \ddot{A}(t)$ were so complicated that we could not obtain any additional information.

One of the main obstacles in pursuing a deeper geometric analysis in 3 were the constraints, so we further tried to obtain equations of motion without constraints. For this, we used the stereographic projection through the north pole of the sphere into the plane of the equator, and succeeded in expressing the problem in terms of coordinates of the curved complex plane endowed with a Riemannian conformal metric denoted by $\mathbb{M}_{R}^{2}$.

In this paper we obtain the equations of motion which generalize the (Euclidian classical) gravitational Newtonian problem by using cotangent potentials and we analyze solutions which are invariant by the action of one subgroup of isometries of $S U(2) /\{ \pm I\}$ of $\mathbb{M}_{R}^{2}$, the so-called relative equilibria of the curved $n$-body problem. We prove that the model studied in [3] and the model studied in this work are equivalent.

Our paper is organized as follows. In Section 2, using the stereographic projection and techniques of differential geometry, we translate the problem from the sphere of radius $R$ to the curved plane $\mathbb{M}_{R}^{2}$ and we introduce complex variables to facilitate the computations. As is customary for this problem, we will use the cotangent potential (see 4 for more details). We obtain the equations of motion for the $n$-body problem by considering the equation of the geodesics in such a space and curving the gradient of the potential with a convenient conformal factor. The original equations for the $n$-body problem on the sphere are derived using variational methods, which require the natural extension of the metric and the differential properties from the embedded sphere to the ambient space $\mathbb{R}^{3}$. The big advantage of the intrinsic approach introduced in this paper is that no such extension is necessary. We end Section 2 by proving that the new potential and its gradient are equivalent to the original potential and its gradient. Then we show that the equations of motion of the $n$-body problem on $\mathbb{M}_{R}^{2}$, and the original equations on the sphere, are equivalent.

In Section 3 we consider a suitable one-dimensional additive subgroup of the Lie algebra $s u(2)$ and, projecting it via the exponential map onto $S U(2)$, we obtain a one-dimensional subgroup of isometries. When this subgroup is associated to the corresponding one-dimensional group of Moebius transformations, we show that with the help of the Principal Axis Theorem the above subgroup generates all the relative equilibria of the $n$-body problem in $\mathbb{M}_{R}^{2}$. We thus obtain several algebraic equations that characterize all relative equilibria.

In Section 4 we study the relative equilibria of the 2 -body problem in $\mathbb{M}_{R}^{2}$. The system describing this problem is not integrable (see [12] and [14] for more details). Therefore the study of relative equilibria for the 2-body problem on $\mathbb{M}_{R}^{2}$ is of relevant importance, since they provide families of periodic orbits in a nonintegrable system. Finally, in Section 5, we study the relative equilibria of the 3-body problem in $\mathbb{M}_{R}^{2}$. We first consider the collinear case, which corresponds to the Eulerian orbits, and 
obtain the necessary and sufficient conditions for their existence. We end the paper with a result that gives a necessary and sufficient condition for the existence of the Lagrangian relative equilibria.

\section{Equations of Motion}

Consider a surface of constant positive curvature $\mathbf{K}$. It is well known (see for instance [7]) that this surface is locally represented by the two-dimensional sphere of radius $R=1 / \sqrt{\mathbf{K}}$.

Let $\Pi$ be the stereographic projection through the north pole into the plane of the equator

$$
\begin{aligned}
\Pi: & S_{R}^{2} \rightarrow \mathbb{R}^{2}, \\
& Q \rightarrow q .
\end{aligned}
$$

If $Q=(x, y, z)$, then $q=(u, v)$, where

$$
u=\frac{R x}{R-z} \quad \text { and } \quad v=\frac{R y}{R-z} .
$$

The inverse function is given by

$$
x=\frac{2 R^{2} u}{u^{2}+v^{2}+R^{2}}, \quad y=\frac{2 R^{2} v}{u^{2}+v^{2}+R^{2}}, \quad z=\frac{R\left(u^{2}+v^{2}-R^{2}\right)}{u^{2}+v^{2}+R^{2}} .
$$

The metric (distance) of the sphere $S_{R}^{2}$ is transformed into the metric

$$
d s^{2}=\frac{4 R^{4}}{\left(u^{2}+v^{2}+R^{2}\right)^{2}}\left(d u^{2}+d v^{2}\right) .
$$

Under the above transformations the geodesics of $S_{R}^{2}$ are mapped as follows: the equator of $S_{R}^{2}$ into the geodesic circle of radius $R$ (the unique circle which is a geodesic in the curved plane), the meridians into straight lines through the origin, and the other great circles of $S_{R}^{2}$ into ellipses. The length for all these geodesics is $2 \pi$. In fact, since the metric is conformal with factor of conformity $\lambda(u, v)=\frac{4 R^{4}}{\left(u^{2}+v^{2}+R^{2}\right)^{2}}$, the Christoffel symbols associated to such a metric are given by

$$
\begin{aligned}
-\Gamma_{22}^{1} & =\Gamma_{11}^{1}=\Gamma_{12}^{2}=\frac{1}{2 \lambda(u, v)} \frac{\partial \lambda}{\partial u}=-\frac{2 u}{\left(u^{2}+v^{2}+R^{2}\right)^{2}}, \\
\Gamma_{22}^{2} & =-\Gamma_{11}^{2}=\Gamma_{12}^{1}=\frac{1}{2 \lambda(u, v)} \frac{\partial \lambda}{\partial v}=-\frac{2 v}{\left(u^{2}+v^{2}+R^{2}\right)^{2}} .
\end{aligned}
$$

Therefore, in these coordinates, the geodesics can also be obtained by solving the system (see 8 , for more details):

$$
\begin{aligned}
& \ddot{u}+\Gamma_{11}^{1} \dot{u}^{2}+2 \Gamma_{12}^{1} \dot{u} \dot{v}+\Gamma_{22}^{1} \dot{v}^{2}=0, \\
& \ddot{v}+\Gamma_{11}^{2} \dot{u}^{2}+2 \Gamma_{12}^{2} \dot{u} \dot{v}+\Gamma_{22}^{2} \dot{v}^{2}=0,
\end{aligned}
$$

which is equivalent to the system of second order differential equations

$$
\begin{aligned}
\ddot{u} & =\frac{2}{R^{2}+u^{2}+v^{2}}\left(u \dot{u}^{2}+2 v \dot{u} \dot{v}-u \dot{v}^{2}\right), \\
\ddot{v} & =\frac{2}{R^{2}+u^{2}+v^{2}}\left(v \dot{v}^{2}+2 u \dot{u} \dot{v}-v \dot{u}^{2}\right) .
\end{aligned}
$$


The plane with the above metric is called a spherical plane, which we denote by $\mathbb{R}_{s p h}^{2}$. The distance between two points $q_{i}, q_{j} \in \mathbb{R}_{s p h}^{2}$ takes the form

$$
d_{k j}=d\left(q_{k}, q_{j}\right)=d\left(Q_{k}, Q_{j}\right)=R \arccos \frac{Q_{k} \cdot Q_{j}}{R^{2}}
$$

with

$$
Q_{k} \cdot Q_{j}=\frac{4 R^{4}\left(q_{k} \cdot q_{j}\right)+R^{2}\left(\left\|q_{k}\right\|^{2}-R^{2}\right)\left(\left\|q_{j}\right\|^{2}-R^{2}\right)}{\left(\left\|q_{k}\right\|^{2}+R^{2}\right)\left(\left\|q_{j}\right\|^{2}+R^{2}\right)},
$$

where $\cdot$ denotes the standard dot product. In order to introduce the cotangent potential, as in [3], we first notice that

$$
\cot _{R}\left(\frac{d_{k j}}{R}\right)=\frac{4 R^{2}\left(q_{k} \cdot q_{j}\right)+\left(\left\|q_{k}\right\|^{2}-R^{2}\right)\left(\left\|q_{j}\right\|^{2}-R^{2}\right)}{W}
$$

where

$$
\begin{aligned}
W & =\sqrt{W_{1}-W_{2}}, \\
W_{1} & =\left(\left\|q_{k}\right\|^{2}+R^{2}\right)^{2}\left(\left\|q_{j}\right\|^{2}+R^{2}\right)^{2}, \quad \text { and } \\
W_{2} & =\left[4 R^{2}\left(q_{k} \cdot q_{j}\right)+\left(\left\|q_{k}\right\|^{2}-R^{2}\right)\left(\left\|q_{j}\right\|^{2}-R^{2}\right)\right]^{2} .
\end{aligned}
$$

To simplify the computations, we further introduce complex variables, $z=u+$ $i v, \bar{z}=u-i v$. We can thus identify $\mathbb{R}_{s p h}^{2}$ with the complex plane $\mathbb{C}$. The distance (2.2) and equation (2.5) take the form

$$
\begin{gathered}
d s^{2}=\frac{4 R^{4} d z d \bar{z}}{\left(R^{2}+|z|^{2}\right)^{2}} \\
\cot _{R}\left(\frac{d_{k j}}{R}\right)=\frac{2\left(z_{k} \bar{z}_{j}+z_{j} \bar{z}_{k}\right) R^{2}+\left(\left|z_{k}\right|^{2}-R^{2}\right)\left(\left|z_{j}\right|^{2}-R^{2}\right)}{\sqrt{\Theta_{1,(k, j)}(z, \bar{z})}},
\end{gathered}
$$

respectively, where

$$
\begin{aligned}
\Theta_{1,(k, j)}(z, \bar{z}) & =\left(\left|z_{k}\right|^{2}+R^{2}\right)^{2}\left(\left|z_{j}\right|^{2}+R^{2}\right)^{2} \\
& -\left[2\left(z_{k} \bar{z}_{j}+z_{j} \bar{z}_{k}\right) R^{2}+\left(\left|z_{k}\right|^{2}-R^{2}\right)\left(\left|z_{j}\right|^{2}-R^{2}\right)\right]^{2} .
\end{aligned}
$$

We denote by $\mathbb{M}_{R}^{2}$ the space $\mathbb{C}$ endowed with the metric (2.6).

2.1. The intrinsic approach. Let $z=\left(z_{1}, z_{2}, \ldots, z_{n}\right) \in \mathbb{C}^{n}$ be the position of $n$ point particles with masses $m_{1}, m_{2}, \ldots, m_{n}>0$ in the the space $\mathbb{M}_{R}^{2}$. We assume that the particles are moving under the action of the Lagrangian

$$
L_{R}(z, \dot{z}, \bar{z}, \dot{\bar{z}})=T_{R}(z, \dot{z}, \bar{z}, \dot{\bar{z}})+U_{R}(z, \bar{z}),
$$

where

$$
T_{R}(z, \dot{z}, \bar{z}, \dot{\bar{z}})=\frac{1}{2} \sum_{k=1}^{n} m_{k} \lambda\left(z_{k}, \bar{z}_{k}\right)\left|\dot{z}_{k}\right|^{2}
$$

is the kinetic energy,

$$
\begin{aligned}
U_{R}(z, \bar{z}) & =\frac{1}{R} \sum_{1 \leq k<j \leq n}^{n} m_{k} m_{j} \cot _{R}\left(\frac{d_{k j}}{R}\right) \\
& =\frac{1}{R} \sum_{1 \leq k<j \leq n}^{n} m_{k} m_{j} \frac{2\left(z_{k} \bar{z}_{j}+z_{j} \bar{z}_{k}\right) R^{2}+\left(\left|z_{k}\right|^{2}-R^{2}\right)\left(\left|z_{j}\right|^{2}-R^{2}\right)}{\sqrt{\Theta_{1,(k, j)}(z, \bar{z})}}
\end{aligned}
$$


is the cotangent force function (i.e. the negative of the potential) defined in the set $\mathbb{M}_{R}^{2 n} \backslash \Delta$, where $\Delta$ is the set of zeros of $\Theta_{1,(k, j)}(z, \bar{z})$, and

$$
\lambda\left(z_{k}, \bar{z}_{k}\right)=\frac{4 R^{4}}{\left(R^{2}+\left|z_{k}\right|^{2}\right)^{2}}
$$

is the conformal function for its Riemannian metric.

The following result gives the equations of motion for the mechanical system defined in $\mathbb{M}_{R}^{2}$.

\section{Lemma 2.1. Let}

$$
L=L_{R}(z, \dot{z}, \bar{z}, \dot{\bar{z}})=\frac{1}{2} \sum_{k=1}^{n} m_{k} \lambda\left(z_{k}, \bar{z}_{k}\right)\left|\dot{z}_{k}\right|^{2}+U_{R}(z, \bar{z})
$$

be the Lagrangian defined in (2.9) for the given problem. Then the curve solution of the corresponding Euler-Lagrange equations satisfies the system of second order ordinary differential equations

$$
m_{k} \ddot{z}_{k}=\frac{2 m_{k} \bar{z}_{k} \dot{z}_{k}^{2}}{R^{2}+\left|z_{k}\right|^{2}}+\frac{2}{\lambda\left(z_{k}, \bar{z}_{k}\right)} \frac{\partial U_{R}}{\partial \bar{z}_{k}}(z, \bar{z})
$$

where

$$
\frac{\partial U_{R}}{\partial \bar{z}_{k}}=\sum_{j=1, j \neq k}^{n} \frac{2 m_{k} m_{j} R P_{1,(k, j)}(z, \bar{z})}{\left[\Theta_{1,(k, j)}(z, \bar{z})\right]^{3 / 2}}
$$

and

$$
\begin{aligned}
P_{1,(k, j)}(z, \bar{z})= & \left(R^{2}+\left|z_{k}\right|^{2}\right)\left(\left|z_{j}\right|^{2}+R^{2}\right)^{2} \\
& {\left[\left(\left|z_{j}\right|^{2}-\left(z_{k} \bar{z}_{j}+z_{j} \bar{z}_{k}\right)-R^{2}\right) z_{k}+\left(\left|z_{k}\right|^{2}+R^{2}\right) z_{j}\right] } \\
= & \left(R^{2}+\left|z_{k}\right|^{2}\right)\left(\left|z_{j}\right|^{2}+R^{2}\right)^{2}\left(R^{2}+\bar{z}_{j} z_{k}\right)\left(z_{j}-z_{k}\right) .
\end{aligned}
$$

Proof. We recall that in complex variables the Euler-Lagrange equations are

$$
\frac{d}{d t}\left(\frac{\partial L}{\partial \dot{\bar{z}}_{k}}\right)=\frac{\partial L}{\partial \bar{z}_{k}}
$$

Therefore,

$$
\frac{\partial L}{\partial \bar{z}_{k}}=\frac{1}{2} m_{k} \frac{\partial \lambda}{\partial \bar{z}_{k}}\left(z_{k}, \bar{z}_{k}\right)\left|\dot{z}_{k}\right|^{2}+\frac{\partial U_{R}}{\partial \bar{z}_{k}}\left(z_{k}, \bar{z}_{k}\right)
$$

and

$$
\frac{d}{d t} \frac{\partial L}{\partial \dot{\bar{z}}_{k}}=\frac{1}{2} m_{k}\left(\frac{\partial \lambda}{\partial z_{k}}\left(z_{k}, \bar{z}_{k}\right) \dot{z}_{k}+\frac{\partial \lambda}{\partial \bar{z}_{k}}\left(z_{k}, \bar{z}_{k}\right) \dot{\bar{z}}_{k}\right) \dot{z}_{k}+\frac{1}{2} m_{k} \lambda\left(z_{k}, \bar{z}_{k}\right) \ddot{z}_{k} .
$$

If we compare equations (2.16) and (2.17) we obtain

$$
m_{k} \frac{\partial \lambda}{\partial z_{k}}\left(z_{k}, \bar{z}_{k}\right) \dot{z}_{k}^{2}+m_{k} \lambda\left(z_{k}, \bar{z}_{k}\right) \ddot{z}_{k}=2 \frac{\partial U_{R}}{\partial \bar{z}_{k}}\left(z_{k}, \bar{z}_{k}\right) .
$$

From equation (2.18) we obtain

$$
\frac{m_{k}}{\lambda\left(z_{k}, \bar{z}_{k}\right)} \frac{\partial \lambda}{\partial z_{k}}\left(z_{k}, \bar{z}_{k}\right) \dot{z}_{k}^{2}+m_{k} \ddot{z}_{k}=\frac{2}{\lambda\left(z_{k}, \bar{z}_{k}\right)} \frac{\partial U_{R}}{\partial \bar{z}_{k}}\left(z_{k}, \bar{z}_{k}\right) .
$$


A straightforward computation shows that

$$
\frac{1}{\lambda\left(z_{k}, \bar{z}_{k}\right)} \frac{\partial \lambda}{\partial z_{k}}\left(z_{k}, \bar{z}_{k}\right)=-\frac{2 \bar{z}_{k}}{R^{2}+\left|z_{k}\right|^{2}} .
$$

The substitution of this expression into equation (2.19) ends the proof.

We can now prove the following result.

Corollary 2.2. If in the space $\mathbb{M}_{R}^{2}$ the potential is zero or is constant in the whole space, then the particles move freely along geodesics.

Proof. From Lemma 2.1, the equations of motion have the form

$$
m_{k} \ddot{z}_{k}+m_{k} \Gamma^{k}\left(\dot{z}_{k}, \dot{\bar{z}}_{k}\right)=\frac{2}{\lambda\left(z_{k}, \bar{z}_{k}\right)} \frac{\partial U_{R}}{\partial \bar{z}_{k}}(z, \bar{z})
$$

The right hand side of (2.20) is the $k$-coordinate of the curved gradient $U_{R}$ with the new metric, and the left hand side is the equation of the geodesics associated to the Riemannian metric when the right hand side vanishes. Here $\left\{\Gamma^{k}\left(\dot{z}_{k}, \dot{\bar{z}}_{k}\right)\right\}$ denotes the associated connection compatible with the corresponding Riemannian metric when it is applied to the velocity vectors $\dot{z}_{k}, \dot{\bar{z}}_{k}$, which is obtained from equations (2.4) by computing

$$
\begin{aligned}
\frac{\ddot{z}_{k}}{2} & =\frac{\left(\ddot{u}_{k}+i \ddot{v}_{k}\right)}{2} \\
& =\frac{1}{R^{2}+u_{k}^{2}+v_{k}^{2}}\left(\left(u_{k}-i v_{k}\right) \dot{u}_{k}^{2}-2\left(v_{k}-i u_{k}\right) v_{k} \dot{u}_{k} \dot{v}_{k}+\left(-u_{k}+i v_{k}\right) \dot{u}_{k}^{2}\right) \\
& =\frac{\left(u_{k}-i v_{k}\right)}{R^{2}+u_{k}^{2}+v_{k}^{2}}\left(\dot{u}_{k}^{2}+2 i v_{k} \dot{u}_{k} \dot{v}_{k}-\dot{u}_{k}^{2}\right)=\frac{\left(u_{k}-i v_{k}\right)}{R^{2}+u_{k}^{2}+v_{k}^{2}}\left(\dot{u}_{k}^{2}+i \dot{u}_{k}^{2}\right)^{2} \\
(2.21) & =\frac{\bar{z}_{k} \dot{z}_{k}^{2}}{R^{2}+\left|z_{k}\right|^{2}},
\end{aligned}
$$

a fact that proves the result.

2.2. Equivalence of the models. In [3] the equations of motion for the $n$-body problem in spaces of constant curvature $\mathbf{K}>0$ are given by

$$
m_{i} \ddot{\mathbf{q}}_{i}=\nabla_{\mathbf{q}_{i}} V_{\mathbf{K}}(\mathbf{q})-m_{i} \mathbf{K}\left(\dot{\mathbf{q}}_{i} \cdot \dot{\mathbf{q}}_{i}\right) \mathbf{q}_{i}, \quad \mathbf{q}_{i} \cdot \mathbf{q}_{i}=\mathbf{K}^{-1}, \quad \begin{array}{r}
\mathbf{K} \neq 0, \\
i=1, \ldots, n,
\end{array}
$$

where we have replaced the original coordinates $Q_{i}$ by $\mathbf{q}_{i}$ and $U_{\kappa}$ by $V_{\mathbf{K}}$.

$$
V_{\mathbf{K}}(\mathbf{q})=\sum_{1 \leq i<j \leq n} \frac{m_{i} m_{j} \mathbf{K}^{1 / 2}\left(\mathbf{K} \mathbf{q}_{i} \cdot \mathbf{q}_{j}\right)}{\left[\left(\mathbf{K} \mathbf{q}_{i} \cdot \mathbf{q}_{i}\right)\left(\mathbf{K} \mathbf{q}_{j} \cdot \mathbf{q}_{j}\right)-\left(\mathbf{K} \mathbf{q}_{i} \cdot \mathbf{q}_{j}\right)^{2}\right]^{1 / 2}}
$$

is the force function, and $\mathbf{q}_{i} \cdot \mathbf{q}_{i}=\mathbf{K}^{-1}$ are the constraints that maintain the particles on the sphere of curvature $\mathbf{K}$. Then

$$
\nabla_{\mathbf{q}_{i}} V_{\mathbf{K}}(\mathbf{q})=\sum_{j=1, j \neq i}^{n} \frac{m_{i} m_{j} \mathbf{K}^{3 / 2}\left(\mathbf{K} \mathbf{q}_{j} \cdot \mathbf{q}_{j}\right)\left[\left(\mathbf{K} \mathbf{q}_{i} \cdot \mathbf{q}_{i}\right) \mathbf{q}_{j}-\left(\mathbf{K} \mathbf{q}_{i} \cdot \mathbf{q}_{j}\right) \mathbf{q}_{i}\right]}{\left[\left(\mathbf{K} \mathbf{q}_{i} \cdot \mathbf{q}_{i}\right)\left(\mathbf{K} \mathbf{q}_{j} \cdot \mathbf{q}_{j}\right)-\left(\mathbf{K} \mathbf{q}_{i} \cdot \mathbf{q}_{j}\right)^{2}\right]^{3 / 2}}
$$

Using the constraint $\mathbf{K} \mathbf{q}_{i} \cdot \mathbf{q}_{i}=1$, it follows that

$$
\nabla_{\mathbf{q}_{i}} V_{\mathbf{K}}(\mathbf{q})=\sum_{j=1, j \neq i}^{n} \frac{m_{i} m_{j} \mathbf{K}^{3 / 2}\left[\mathbf{q}_{j}-\left(\mathbf{K q}_{i} \cdot \mathbf{q}_{j}\right) \mathbf{q}_{i}\right]}{\left[1-\left(\mathbf{K} \mathbf{q}_{i} \cdot \mathbf{q}_{j}\right)^{2}\right]^{3 / 2}}
$$


Equations (2.22) have the total energy integral

$$
H_{\mathbf{K}}(\mathbf{q}, \mathbf{p})=T_{\mathbf{K}}(\mathbf{q}, \mathbf{p})-V_{\mathbf{K}}(\mathbf{q}),
$$

where $T_{\mathbf{K}}(\mathbf{q}, \mathbf{p}):=\frac{1}{2} \sum_{i=1}^{n} m_{i}^{-1}\left(\mathbf{p}_{i} \cdot \mathbf{p}_{i}\right)\left(\mathbf{K} \mathbf{q}_{i} \cdot \mathbf{q}_{i}\right)$ is the kinetic energy and $\mathbf{p}$ $:=\left(\mathbf{p}_{1}, \ldots, \mathbf{p}_{n}\right)$ denotes the momentum of the $n$-body system, with $\mathbf{p}_{i}:=m_{i} \dot{\mathbf{q}}_{i}$ representing the momentum of the body of mass $m_{i}, i=1, \ldots, n$.

Returning to our equations on $\mathbb{M}_{R}^{2}$, it is easy to verify that system (2.20) has the total energy integral

$$
H_{R}(z, \dot{z})=\frac{1}{2} \sum_{k=1}^{n} m_{k} \lambda\left(z_{k}, \bar{z}_{k}\right)\left|\dot{z}_{k}\right|^{2}-U_{R}\left(z_{k}, \bar{z}_{k}\right)
$$

Now we can prove the equivalence between the equations of motion of the $n-$ body problem defined on the sphere $S_{\mathbf{K}}^{2}$ of curvature $\mathbf{K}>0$ and the equations we have obtained on $\mathbb{M}_{R}^{2}$.

Theorem 2.3. The equations of motion for the $n$-body problem on $\mathbb{M}_{R}^{2}$, given by system (2.20), and the corresponding equations on the sphere $S_{\mathbf{K}}^{2}$, given by system (2.22), are equivalent.

Remark. In [3], the equations of motion were obtained using constrained Lagrangian dynamics. This variational method required the extension of the metric and the differential properties from the sphere to the ambient space. Consequently, in the above form of $V_{\mathbf{K}}$, the expressions $\mathbf{K q}_{i} \cdot \mathbf{q}_{i}$ occur explicitly, though each of them takes the value 1 . In our intrinsic approach, however, we don't need to extend the force function to any ambient space. In fact this is the big advantage of working directly in a two-dimensional space instead of embedding the problem in a higherdimensional space with constraints, so we can impose the condition $\mathbf{K q}_{i} \cdot \mathbf{q}_{i}=1$ from the very beginning, i.e. work with

$$
V_{\mathbf{K}}(\mathbf{q})=\sum_{1 \leq i<j \leq n} \frac{m_{i} m_{j} \mathbf{K}^{1 / 2}\left(\mathbf{K} \mathbf{q}_{i} \cdot \mathbf{q}_{j}\right)}{\left[1-\left(\mathbf{K q}_{i} \cdot \mathbf{q}_{j}\right)^{2}\right]^{1 / 2}}
$$

In [3], however, this early substitution would lead to the incorrect gradient

$$
\nabla_{\mathbf{q}_{i}} V_{\mathbf{K}}(\mathbf{q})=\sum_{\substack{j=1 \\ j \neq i}}^{n} \frac{m_{i} m_{j} \mathbf{K}^{3 / 2} \mathbf{q}_{j}}{\left[1-\left(\mathbf{K q}_{i} \cdot \mathbf{q}_{j}\right)^{2}\right]^{3 / 2}}
$$

which is not the same as (2.23). Thus the question arises as to whether our approach, which starts from (2.26), leads to a gradient equivalent to (2.23). This is why, in the proof below, we will show not only the equivalence of the Hamiltonians (2.24) and (2.25), but also of the gradients for the corresponding force functions.

Proof of Theorem 2.3. A direct computation shows that the inner product $\mathbf{q}_{k} \cdot \mathbf{q}_{j}$ in complex variables takes the form

$$
\mathbf{q}_{k} \cdot \mathbf{q}_{j}=\frac{2 R^{4}\left(z_{k} \bar{z}_{j}+z_{j} \bar{z}_{k}\right)+R^{2}\left(\left|z_{k}\right|^{2}-R^{2}\right)\left(\left|z_{j}\right|^{2}-R^{2}\right)}{\left(\left|z_{k}\right|^{2}+R^{2}\right)\left(\left|z_{j}\right|^{2}+R^{2}\right)} .
$$


Using this fact and the stereographic projection, we can write the force function $V_{\mathbf{K}}(\mathbf{q})$ in complex coordinates as

$$
\begin{aligned}
V_{\mathbf{K}}(\mathbf{q}) & =\sum_{j=1, j \neq k}^{n} \frac{m_{k} m_{j} \mathbf{K}^{1 / 2}\left(\mathbf{K} \mathbf{q}_{k} \cdot \mathbf{q}_{j}\right)}{\left[1-\left(\mathbf{K} \mathbf{q}_{k} \cdot \mathbf{q}_{j}\right)^{2}\right]^{1 / 2}} \\
& =\sum_{j=1, j \neq k}^{n} \frac{m_{k} m_{j} \frac{1}{R}\left[\frac{1}{R^{2}} \mathbf{q}_{k} \cdot \mathbf{q}_{j}\right]}{\left[1-\left(\frac{1}{R^{2}} \mathbf{q}_{k} \cdot \mathbf{q}_{j}\right)^{2}\right]^{1 / 2}}=\frac{1}{R} \sum_{j=1, j \neq k}^{n} \frac{m_{k} m_{j} \mathbf{q}_{k} \cdot \mathbf{q}_{j}}{\left[R^{4}-\left(\mathbf{q}_{k} \cdot \mathbf{q}_{j}\right)^{2}\right]^{1 / 2}} \\
& =\frac{1}{R} \sum_{j=1, j \neq k}^{n} m_{k} m_{j} \frac{2\left(z_{k} \bar{z}_{j}+z_{j} \bar{z}_{k}\right) R^{2}+\left(\left|z_{k}\right|^{2}-R^{2}\right)\left(\left|z_{j}\right|^{2}-R^{2}\right)}{\sqrt{\Theta_{1,(k, j)}(z, \bar{z})}} \\
& =U_{R}(z, \bar{z}),
\end{aligned}
$$

where $\Theta_{1,(k, j)}(z, \bar{z})$ is defined in (2.8). Thus the force function $V_{\mathbf{K}}(\mathbf{q})$ and $U_{R}(z, \bar{z})$ are identical.

To show that their gradients are identical, notice that since $\frac{\partial \mathbf{q}_{j}}{\partial \bar{z}_{k}}$ vanishes if $j \neq k$, the gradient (2.23) takes in complex variables the form

$$
\begin{aligned}
\frac{\partial V_{\mathbf{K}}}{\partial \bar{z}_{k}}(\mathbf{q}) & =\sum_{j=1}^{n} \nabla_{\mathbf{q}_{j}} V_{\mathbf{K}}(\mathbf{q}) \cdot \frac{\partial \mathbf{q}_{j}}{\partial \bar{z}_{k}} \\
& =\left(\sum_{j=1, j \neq k}^{n} \frac{m_{j} m_{k}(\mathbf{K})^{3 / 2}\left[\mathbf{q}_{j}-\left(\mathbf{K} \mathbf{q}_{k} \cdot \mathbf{q}_{j}\right) \mathbf{q}_{k}\right]}{\left[1-\left(\mathbf{K} \mathbf{q}_{k} \cdot \mathbf{q}_{j}\right)^{2}\right]^{3 / 2}}\right) \cdot \frac{\partial \mathbf{q}_{k}}{\partial \bar{z}_{k}} .
\end{aligned}
$$

For any $j=1,2, \ldots, n$, the position $\mathbf{q}_{j}$ on the sphere can be written in complex coordinates as

$$
\mathbf{q}_{j}=\left(\frac{R^{2}\left(z_{j}+\bar{z}_{j}\right)}{z_{j} \bar{z}_{j}+R^{2}}, \frac{-i R^{2}\left(z_{j}-\bar{z}_{j}\right)}{z_{j} \bar{z}_{j}+R^{2}}, \frac{R\left(z_{j} \bar{z}_{j}-R^{2}\right)}{z_{j} \bar{z}_{j}+R^{2}}\right) .
$$

Therefore, for $j=k$, we obtain

$$
\frac{\partial \mathbf{q}_{k}}{\partial \bar{z}_{k}}=\frac{R^{2}}{\left(\left|z_{k}\right|^{2}+R^{2}\right)^{2}}\left(-z_{k}^{2}+R^{2}, i\left(z_{k}^{2}+R^{2}\right), 2 R z_{k}\right),
$$

and this implies that

$$
\mathbf{q}_{j} \cdot \frac{\partial \mathbf{q}_{k}}{\partial \bar{z}_{k}}=\frac{2 R^{4}\left(\left(\left|z_{j}\right|^{2}-\left(z_{k} \bar{z}_{j}+z_{j} \bar{z}_{k}\right)-R^{2}\right) z_{k}+\left(\left|z_{k}\right|^{2}+R^{2}\right) z_{j}\right)}{\left(\left|z_{j}\right|^{2}+R^{2}\right)\left(\left|z_{k}\right|^{2}+R^{2}\right)^{2}} .
$$

From the last equality, we have that $\mathbf{q}_{k} \cdot \frac{\partial \mathbf{q}_{k}}{\partial \bar{z}_{k}}=0$. Using this identity in equation (2.28), we get

$$
\begin{aligned}
\frac{\partial V_{\mathbf{K}}}{\partial \bar{z}_{k}}(\mathbf{q}) & =\sum_{j=1, j \neq k}^{n} \frac{m_{j} m_{k}(\mathbf{K})^{3 / 2}}{\left[1-\left(\mathbf{K} \mathbf{q}_{k} \cdot \mathbf{q}_{j}\right)^{2}\right]^{3 / 2}}\left[\left[\mathbf{q}_{j}-\left(\mathbf{K} \mathbf{q}_{k} \cdot \mathbf{q}_{j}\right) \mathbf{q}_{k}\right] \cdot \frac{\partial \mathbf{q}_{k}}{\partial \bar{z}_{k}}\right] \\
& =\sum_{j=1, j \neq k}^{n} \frac{m_{j} m_{k}(\mathbf{K})^{3 / 2}}{\left[1-\left(\mathbf{K} \mathbf{q}_{k} \cdot \mathbf{q}_{j}\right)^{2}\right]^{3 / 2}}\left(\mathbf{q}_{j} \cdot \frac{\partial \mathbf{q}_{k}}{\partial \bar{z}_{k}}\right) .
\end{aligned}
$$


In complex variables, equation (2.30) becomes

$$
\begin{aligned}
\frac{\partial V_{\mathbf{K}}}{\partial \bar{z}_{k}}(\mathbf{q}) & =\sum_{j=1, j \neq k}^{n} \frac{m_{j} m_{k}(\mathbf{K})^{3 / 2}}{\left[1-\left(\mathbf{K q}_{k} \cdot \mathbf{q}_{j}\right)^{2}\right]^{3 / 2}}\left(\mathbf{q}_{j} \cdot \frac{\partial \mathbf{q}_{k}}{\partial \bar{z}_{k}}\right) \\
& =\sum_{j=1, j \neq k}^{n} m_{j} m_{k} \frac{2 R\left(\left|z_{j}\right|^{2}+R^{2}\right)^{2}\left(\left|z_{k}\right|+R^{2}\right)^{2} L_{1,(k, j)}(z, \bar{z})}{\left[\Theta_{1,(k, j)}(z, \bar{z})\right]^{3 / 2}} \\
& =\frac{\partial U_{R}}{\partial \bar{z}_{k}}(z, \bar{z}),
\end{aligned}
$$

where $L_{1,(k, j)}(z, \bar{z})=\left(\left|z_{j}\right|^{2}-\left(z_{k} \bar{z}_{j}+z_{j} \bar{z}_{k}\right)-R^{2}\right) z_{k}+\left(\left|z_{k}\right|^{2}+R^{2}\right) z_{j}$ and $\Theta_{1,(k, j)}(z, \bar{z})$ is defined in (2.8).

Equations (2.27) and (2.31) prove the equivalence of the force functions, on one hand, and of their gradients, on the other hand. To finish the proof, we still need to show that the kinetic energies are equivalent.

Since $\Pi$ is an isometry, it follows that

$$
\dot{\mathbf{q}}_{k} \cdot \dot{\mathbf{q}}_{k}=\lambda\left(z_{k}, \bar{z}_{k}\right)\left|\dot{z}_{k}\right|^{2} .
$$

From equation (2.29), the coordinates of the velocity $\dot{\mathbf{q}}_{k}$ are

$$
\begin{aligned}
& \frac{R^{2} \dot{z}_{k}\left(R^{2}-\bar{z}_{k}^{2}\right)+\dot{\bar{z}}_{k}\left(R^{2}-z_{k}^{2}\right)}{\left(z_{k} \bar{z}_{k}+R^{2}\right)^{2}}, \frac{-i R^{2}\left(\dot{z}_{k}\left(R^{2}+\bar{z}_{k}^{2}\right)-\dot{\bar{z}}_{k}\left(R^{2}+z_{k}^{2}\right)\right)}{\left(z_{k} \bar{z}_{k}+R^{2}\right)^{2}}, \\
& \text { and } \frac{R^{3}\left(\dot{z}_{k} \bar{z}_{k}+z_{k} \dot{\bar{z}}_{k}\right)}{\left(z_{k} \bar{z}_{k}+R^{2}\right)^{2}} .
\end{aligned}
$$

By straightforward computations we get

$$
\begin{aligned}
& T_{\mathbf{K}}(\mathbf{q}, \mathbf{p})=\frac{1}{2} \sum_{k=1}^{n} m_{i}^{-1}\left(\mathbf{p}_{k} \cdot \mathbf{p}_{k}\right)\left(\mathbf{K} \mathbf{q}_{k} \cdot \mathbf{q}_{k}\right)=\frac{1}{2} \sum_{k=1}^{n} m_{k} \dot{\mathbf{q}}_{k} \cdot \dot{\mathbf{q}}_{k} \\
= & \frac{1}{2} \sum_{k=1}^{n} m_{k} \frac{4 R^{2}}{\left(R^{2}+\left|\dot{z}_{k}\right|^{2}\right)^{4}}\left[\left(\dot{z}_{k} R^{2}-\dot{\bar{z}}_{k} z_{k}^{2}\right)\left(\dot{\bar{z}}_{k} R^{2}-\dot{z}_{k} \bar{z}_{k}^{2}\right)+R^{2}\left(\dot{z}_{k} \bar{z}_{k}+z_{k} \dot{\bar{z}}_{k}\right)^{2}\right] \\
= & \frac{1}{2} \sum_{k=1}^{n} m_{k} \frac{4 R^{2} \dot{z}_{k} \dot{\bar{z}}_{k}}{\left(R^{2}+\left|\dot{z}_{k}\right|^{2}\right)^{4}}\left[R^{4}+2 z_{k} \bar{z}_{k}+z_{k}^{2} \bar{z}_{k}^{2}\right] \\
= & \frac{1}{2} \sum_{k=1}^{n} m_{k} \frac{4 R^{2} \dot{z}_{k} \dot{\bar{z}}_{k}}{\left(R^{2}+\left|\dot{z}_{k}\right|^{2}\right)^{2}}\left|\dot{z}_{k}\right|^{2}=\frac{1}{2} \sum_{k=1}^{n} m_{k} \lambda\left(z_{k}, \bar{z}_{k}\right)\left|\dot{z}_{k}\right|^{2}=T_{\mathbf{R}}(\mathbf{z}, \dot{\mathbf{z}}, \overline{\mathbf{z}}, \dot{\bar{z}}) .
\end{aligned}
$$

We thus obtain that the expression for the above kinetic energy and the kinetic energy given in (2.10) are equivalent. Therefore systems (2.20) and (2.22) describe similar qualitative dynamics.

\section{Relative equilibria}

In this section we start the analysis of the dynamics of the particles in $\mathbb{M}_{R}^{2}$, present some general aspects of this problem, and characterize all relative equilibria.

3.1. The singularities in $\mathbb{M}_{R}^{2}$. The singular set in $\mathbb{M}_{R}^{2}$ for the $n$-body problem is the zero set of the equation (2.8):

$$
\Theta_{1,(k, j)}(z, \bar{z})=0 .
$$


The solution of the above equation gives us the following sets:

(1) The singular collision set given by $\Delta^{+}=\bigcup_{k j} \Delta_{k j}^{+}$, where

$$
\Delta_{k j}^{+}=\left\{z_{k}=z_{j}\right\} \text {. }
$$

(2) The singular antipodal set given by $\Delta^{-}=\bigcup_{k j} \Delta_{k j}^{-}$, where

$$
\Delta_{k j}^{-}=\left\{z_{k}=\frac{-R^{2}}{\left|z_{j}\right|^{2}} z_{j}\right\}
$$

We define the total singular set of the problem as

$$
\Delta=\Delta^{+} \cup \Delta^{-} .
$$

The behavior of the motion close to the total singular set for the $n$-body problem in spaces of nonzero Gaussian constant curvature has been widely studied by Florin Diacu 4, who obtained some important results in the intersection $\Delta=\Delta^{+} \cap \Delta^{-}$. He thus showed that there exist solutions that end in hybrid collision-antipodal configurations, solutions that can never reach such a configuration, no matter how close to them the initial conditions are taken, and solutions that remain analytic at such configurations. Moreover, he generalized to the curved case some results that Carl Sundman and Paul Painlevé proved for the Euclidean case.

3.2. Relative equilibria in $\mathbb{M}_{R}^{2}$. Let Iso $\left(\mathbb{M}_{R}^{2}\right)$ be the group of isometries of $\mathbb{M}_{R}^{2}$, and let us denote by $\{G(t)\}$ a one-parametric subgroup of $\operatorname{Iso}\left(\mathbb{M}_{R}^{2}\right)$, which acts coordinatewise in $\mathbb{M}_{R}^{2 n} \backslash \Delta$ and in $\Delta$ leaving them invariant.

Definition 3.1. A relative equilibrium of the curved $n$-body problem is a solution $z(t)$ of $(2.20)$ which is invariant relative to the subgroup $\{G(t)\}$. In other words, the function obtained by the action denoted by $w(t)=G(t) z(t)$ is also a solution of (2.20).

Now we give conditions for the existence of relative equilibria on $\mathbb{M}_{R}^{2}$. Rewriting the equations of motion (2.20) we obtain

$$
m_{k} \ddot{z}_{k}=\frac{2 m_{k} \bar{z}_{k} \dot{z}_{k}^{2}}{R^{2}+\left|z_{k}\right|^{2}}+\frac{\left(R^{2}+\left|z_{k}\right|^{2}\right)^{2}}{2 R^{4}} \frac{\partial U_{R}}{\partial \bar{z}_{k}} .
$$

We know that the group of proper isometries of $\mathbb{M}_{R}^{2}$ is the quotient $\mathrm{SU}(2) /\{ \pm I\}$ of the special unitary subgroup

$$
\mathrm{SU}(2)=\left\{A \in \mathrm{GL}(2, \mathbb{C}) \mid \bar{A}^{T} A=I\right\},
$$

where each matrix $A \in \mathrm{SU}(2)$ has the form

$$
A=\left(\begin{array}{cc}
a & b \\
-\bar{b} & \bar{a}
\end{array}\right),
$$

with $a, b \in \mathbb{C}$ satisfying $|a|^{2}+|b|^{2}=1$, which makes $\mathrm{SU}(2)$ diffeomorphic to one real three-dimensional sphere in $\mathbb{C}^{2}$ (see for instance Doubrovin et al. 8).

Every class $A \in \mathrm{SU}(2) /\{ \pm I\}$ is associated to a unique Moebius transformation (fractional linear transformation) $f_{A}: \mathbb{M}_{R}^{2} \rightarrow \mathbb{M}_{R}^{2}$,

$$
f_{A}(z)=\frac{a z+b}{-\bar{b} z+\bar{a}}
$$

because $f_{A}=f_{-A}$. The Lie algebra of $\mathrm{SU}(2)$ is the three-dimensional real linear space

$$
\operatorname{su}(2)=\left\{X \in \mathrm{M}(2, \mathbb{C}) \mid \bar{X}^{T}=-X, \operatorname{trace}(X)=0\right\}
$$


spanned by the basis of complex Pauli matrices,

$$
\left\{h_{1}=\frac{1}{2}\left(\begin{array}{cc}
0 & 1 \\
-1 & 0
\end{array}\right), \quad h_{2}=\frac{1}{2}\left(\begin{array}{cc}
i & 0 \\
0 & -i
\end{array}\right), \quad h_{3}=\frac{1}{2}\left(\begin{array}{cc}
0 & i \\
i & 0
\end{array}\right)\right\} .
$$

Let us consider the standard exponential map of matrices from the Lie algebra into its Lie group

$$
\exp : \mathrm{su}(2) \rightarrow \mathrm{SU}(2),
$$

applied to the one-parametric additive subgroups (straight lines in $\mathrm{su}(2))\left\{t h_{1}\right\}$, $\left\{t h_{2}\right\}$, and $\left\{t h_{3}\right\}$, for obtaining the respective one-parametric subgroups of $\mathrm{SU}(2)$ :

(1) The subgroup

$$
H_{1}(t)=\exp \left(t h_{1}\right)=\left(\begin{array}{cc}
\cos (t / 2) & \sin (t / 2) \\
-\sin (t / 2) & \cos (t / 2)
\end{array}\right)
$$

which defines the one-parametric family of acting Moebius transformations

$$
f_{H_{1}}(z)=\frac{z \cos (t / 2)+\sin (t / 2)}{-z \sin (t / 2)+\cos (t / 2)}
$$

(2) The subgroup

$$
H_{2}(t)=\exp \left(t h_{2}\right)=\left(\begin{array}{cc}
e^{i t / 2} & 0 \\
0 & e^{-i t / 2}
\end{array}\right),
$$

which defines the one-parametric family of acting Moebius transformations

$$
f_{H_{2}}(z)=e^{i t} z \text {. }
$$

(3) The subgroup

$$
H_{3}(t)=\exp \left(t h_{3}\right)=\left(\begin{array}{cc}
\cos (t / 2) & i \sin (t / 2) \\
i \sin (t / 2) & \cos (t / 2)
\end{array}\right)
$$

which defines the one-parametric family of acting Moebius transformations

$$
f_{H_{3}}(z)=\frac{z \cos (t / 2)+i \sin (t / 2)}{z i \sin (t / 2)+\cos (t / 2)} .
$$

We remark that the rotation group matrix $A(t) \in S O(3)$ introduced at the beginning of this paper (equation (1.1)) is the matrix solution (isometric flow) for the Killing vector field $L_{Z}(x, y, z)=(y,-x, 0)$, which is a rotation around the $z-$ axis defined in the whole $\mathbb{R}^{3}$ that leaves invariant any sphere $S^{2}$ centered at the origin.

Proposition 3.2. If $G: S U(2) / \pm I \rightarrow S O(3)$ is the isomorphism between the above groups of isometries of the sphere, then $G\left(H_{2}(t)\right)=A(t)$.

Proof. By the stereographic projection

$$
\Pi(x, y, z)=\left(\frac{R x}{R-z}, \frac{R y}{R-z}\right),
$$

we can see that since the rotation tangent vector at $(x, y, z)$ in $S_{R}^{2}$ is $(y,-x, 0)$, after the projection we have

$$
D \Pi\left(L_{Z}\right)(x, y, z)^{t}=\left(\begin{array}{ccc}
\frac{R}{R-z} & 0 & \frac{R x}{(R-z)^{2}} \\
0 & \frac{R}{R-z} & \frac{R y}{(R-z)^{2}}
\end{array}\right)\left(\begin{array}{c}
y \\
-x \\
0
\end{array}\right)=\left(\begin{array}{c}
\frac{R y}{R-z} \\
\frac{-R x}{R-z}
\end{array}\right),
$$


which in complex notation corresponds to

$$
v-i u=-i(u+i v)=-i z .
$$

We are thus led to the complex differential equation

$$
\dot{z}=-i z,
$$

which generates the flow $f_{t}(z)=e^{-i t} z$, associated with the one-parametric subgroup of Moebius transformations $f_{H_{2}}(t)$. That is $G\left(H_{2}(t)\right)=A(t)$.

Remark. By the Principal Axis Theorem, which states that any rotation in $\mathbb{R}^{3}$ is around any fixed axis, we can choose without loss of generality the $z$-axis as the rotation axis which corresponds under the isomorphism $G$ to the subgroup $H_{2}$. Actually the subgroups $H_{1}$ and $H_{3}$ represent rotations under $G$ around the $x$ and $y$-axis, respectively, which, when are applied in $\mathbf{M}_{R}^{2}$ with the metric derived from the stereographic projection, become quite complicated. Thanks to the Principal Axis Theorem, we can ignore them, so from here on we will use only the subgroup $\mathrm{H}_{2}$.

To obtain all relative equilibria in $\mathbb{M}_{R}^{2}$, by Proposition 3.2 and the above Remark, we must analyze just the second class of Moebius transformations $\left\{H_{2}(t)\right\}$ given by

$$
w_{k}(t)=e^{i t} z_{k}(t),
$$

where $z(t)=\left(z_{1}(t), \ldots, z_{n}(t)\right)$ is a solution of equation (3.4). So we shall find conditions which guarantee that the solution $z(t)$ of equation (3.7) is also a solution of system (3.4). Straightforward computations lead us to

$$
\begin{aligned}
\dot{w}_{k} & =\left(i z_{k}+\dot{z}_{k}\right) e^{i t} \\
\ddot{w}_{k} & =\left(\ddot{z}_{k}+2 i \dot{z}_{k}-z_{k}\right) e^{i t} \\
\frac{d \bar{z}_{k}}{d \bar{w}_{k}} & =e^{i t} .
\end{aligned}
$$

Therefore $w=w(t)$ is a solution of equation (3.4) if

$$
m_{k} \ddot{w}_{k}=\frac{2 m_{k} \bar{w}_{k} \dot{w}_{k}^{2}}{R^{2}+\left|w_{k}\right|^{2}}+\frac{\left(R^{2}+\left|w_{k}\right|^{2}\right)^{2}}{2 R^{4}} \frac{\partial U_{R}}{\partial \bar{w}_{k}} .
$$

In terms of $z_{k}$, this condition can be written as

$$
\begin{aligned}
m_{k}\left(\ddot{z}_{k}+2 i \dot{z}_{k}-z_{k}\right) e^{i t} & =\frac{2 m_{k} \bar{z}_{k} e^{-i t}\left(i z_{k}+\dot{z}_{k}\right)^{2} e^{2 i t}}{R^{2}+\left|z_{k}\right|^{2}} \\
& +\frac{\left(R^{2}+\left|z_{k}\right|^{2}\right)^{2}}{2 R^{4}} \frac{\partial U_{R}}{\partial \bar{z}_{k}} \frac{d \bar{z}_{k}}{d \bar{w}_{k}} \\
& =\frac{2 m_{k} \bar{z}_{k}\left(i z_{k}+\dot{z}_{k}\right)^{2} e^{i t}}{R^{2}+\left|z_{k}\right|^{2}}+\frac{\left(R^{2}+\left|z_{k}\right|^{2}\right)^{2}}{2 R^{4}} \frac{\partial U_{R}}{\partial \bar{z}_{k}} e^{i t} .
\end{aligned}
$$

Since $z=z(t)$ is a solution of (3.4),$m_{k} \neq 0$, and $e^{i t} \neq 0$, the last relation becomes

$$
2 i \dot{z}_{k}-z_{k}=\frac{2 \bar{z}_{k}\left(2 i z_{k} \dot{z}_{k}-z_{k}^{2}\right)}{R^{2}+\left|z_{k}\right|^{2}},
$$

which is equivalent to

$$
2 i\left[1-\frac{2\left|z_{k}\right|^{2}}{R^{2}+\left|z_{k}\right|^{2}}\right] \dot{z}_{k}=\left[1-\frac{2\left|z_{k}\right|^{2}}{R^{2}+\left|z_{k}\right|^{2}}\right] z_{k} .
$$


Equation (3.10) holds if and only if

$$
1-\frac{2\left|z_{k}\right|^{2}}{R^{2}+\left|z_{k}\right|^{2}}=0
$$

or

$$
2 i \dot{z}_{k}=z_{k} \text {. }
$$

A necessary and sufficient condition for obtaining a relative equilibrium from one fixed point is that all the particles are located on the geodesic circle. We have thus recovered the following result, which was first proved in Diacu et al. [3].

Proposition 3.3. If on the geodesic circle we place $n$ particles with arbitrary masses (avoiding antipodal positions) such that the configuration is a fixed point of the system, then the orbit generated by the action of $\mathrm{H}_{2}(t)$ is a relative equilibrium.

Proof. Condition (3.11) is equivalent to $\left|z_{k}(t)\right|=R$, that is, the $k$-th particle is moving along the geodesic circle. In fact, if all the particles with arbitrary masses move along such geodesics, then the left hand side of equation (2.20) vanishes because it corresponds to a geodesic, so the potential does not act; that is, along this circle, $\frac{\partial U_{R}}{\partial \bar{z}_{k}}(z, \bar{z})=0$. If additionally the particles form a fixed point, that is, if the velocities of the particles vanish $\left(\dot{z}_{i}(t)=0\right)$, then under the action of $\mathrm{H}_{2}$ such a configuration is a relative equilibrium.

Remark. In general, the existence of the above relative equilibria generated by fixed points is an open question.

For $n=3$, however, an affirmative answer has been recently given in [6].

Condition (3.12) holds for $\left|z_{k}(t)\right|=r_{k}$, where $r_{k}$ is a positive number with $r_{k} \neq R$ and $0 \leq r_{k}<\pi R$. We are interested in the study of relative equilibria with at least one particle not on the geodesic circle.

We have the following result which characterizes, modulo isometries, all relative equilibria.

Theorem 3.4. Consider $n$ point particles with masses $m_{1}, m_{2}, \ldots, m_{n}>0$ moving in $\mathbb{M}_{R}^{2}$. A necessary and sufficient condition for the solution

$$
z(t)=\left(z_{1}(t), z_{2}(t), \ldots, z_{n}(t)\right)
$$

of (2.20) to be a relative equilibrium is that the coordinates satisfy the following system given by the rational functions:

$$
\frac{R^{3}\left(R^{2}-r_{k}^{2}\right) z_{k}}{4\left(R^{2}+r_{k}^{2}\right)^{4}}=-\sum_{j=1, j \neq k}^{n} \frac{m_{j}\left(r_{j}^{2}+R^{2}\right)^{2}\left(R^{2}+z_{k} \bar{z}_{j}\right)\left(z_{j}-z_{k}\right)}{\left[\tilde{\Theta}_{1,(k, j)}(z, \bar{z})\right]^{3 / 2}}
$$

where

$$
\tilde{\Theta}_{1,(k, j)}(z, \bar{z})=\left(r_{k}^{2}+R^{2}\right)^{2}\left(r_{j}^{2}+R^{2}\right)^{2}-\left[2\left(z_{k} \bar{z}_{j}+z_{j} \bar{z}_{k}\right) R^{2}+\left(r_{k}^{2}-R^{2}\right)\left(r_{j}^{2}-R^{2}\right)\right]^{2}
$$

and $\left|z_{l}(t)\right|=r_{l} \in[0, \pi R)$.

Proof. A necessary condition for the existence of a relative equilibrium is that the bodies are moving along ordinary circles centered at the origin of the coordinate system in $\mathbb{M}_{R}^{2}$. In order to find all relative equilibria we derive and use condition (3.12), getting

$$
-4 \ddot{z}_{k}=z_{k}
$$


Therefore, substituting (3.14) into equation (3.4) and again using (3.12), we obtain that the coordinates of the relative equilibrium $z=z(t)$ must satisfy the system of fractional algebraic equations

$$
m_{k} z_{k}=\frac{2 m_{k}\left|z_{k}\right|^{2} z_{k}}{R^{2}+\left|z_{k}\right|^{2}}-\frac{2\left(R^{2}+\left|z_{k}\right|^{2}\right)^{2}}{R^{4}} \frac{\partial U_{R}}{\partial \bar{z}_{k}} .
$$

Introducing $\left|z_{l}(t)\right|=r_{l}$ into equation (3.15) and computing the partial derivatives of the potential $U_{R}$, we obtain a system of $n$ equations given by the rational functions (3.13).

Notice that, in order to provide relative equilibria, equation (3.12) imposes a condition on the velocities of equation (3.4).

\section{THE CASE $n=2$}

The two-body problem in spaces of positive Gaussian curvature has been widely studied by several authors; see for instance [1, 2, 10, 11, 13. The study of this problem is divided into two cases. The first case, called the Kepler problem in spaces of positive curvature, assumes that one particle is fixed at the north pole of the two sphere while the second body is moving; this problem is integrable. In the second case both bodies move freely on the sphere. Then, since the center of mass is not a first integral, the problem is not integrable (see [12, 14 for more details).

Therefore the study of relative equilibria for the two-body problem on $\mathbb{M}_{R}^{2}$, that is, the orbits invariant by the action of the one-parametric subgroup $\left\{H_{2}(t)\right\}_{t \in \mathbb{R}}$, is of relevant importance since the set of relative equilibria that we find gives us families of periodic orbits in a nonintegrable system.

For a system of two point particles on $\mathbb{M}_{R}^{2}$ with masses $m_{1}$ and $m_{2}$, the system of algebraic equations (3.13) becomes

$$
\begin{aligned}
& \frac{R^{3}\left(R^{2}-r_{1}^{2}\right) z_{1}}{4\left(R^{2}+r_{1}^{2}\right)^{4}}=-\frac{m_{2}\left(r_{2}^{2}+R^{2}\right)^{2}\left(R^{2}+z_{1} \bar{z}_{2}\right)\left(z_{2}-z_{1}\right)}{\left[\left(\tilde{Q}_{1,(1,2)}(z, \bar{z})\right]^{3 / 2}\right.}, \\
& \frac{R^{3}\left(R^{2}-r_{2}^{2}\right) z_{2}}{4\left(R^{2}+r_{2}^{2}\right)^{4}}=-\frac{m_{1}\left(r_{1}^{2}+R^{2}\right)^{2}\left(R^{2}+z_{2} \bar{z}_{1}\right)\left(z_{1}-z_{2}\right)}{\left[\tilde{Q}_{1,(2,1)}(z, \bar{z})\right]^{3 / 2}},
\end{aligned}
$$

where

$$
\tilde{\Theta}_{1,(k, j)}(z, \bar{z})=\left(r_{1}^{2}+R^{2}\right)^{2}\left(r_{2}^{2}+R^{2}\right)^{2}-\left[2\left(z_{1} \overline{z_{2}}+z_{2} \overline{z_{1}}\right) R^{2}+\left(r_{1}^{2}-R^{2}\right)\left(r_{2}^{2}-R^{2}\right)\right]^{2} .
$$

After some algebraic manipulations, system (4.1) takes the form

$$
\begin{aligned}
\frac{\left(R^{2}-r_{1}^{2}\right)\left(R^{2}+r_{2}^{2}\right)^{2} m_{1}}{\left(R^{2}-r_{2}^{2}\right)\left(R^{2}+r_{1}^{2}\right)^{2} m_{2}} & =\frac{z_{2}\left(R^{2}+z_{1} \bar{z}_{2}\right)\left(z_{2}-z_{1}\right)}{z_{1}\left(R^{2}+z_{2} \bar{z}_{1}\right)\left(z_{1}-z_{2}\right)} \\
& =-\frac{\left(r_{2}^{2} z_{1}+R^{2} z_{2}\right)\left(z_{1}-z_{2}\right)}{\left(r_{1}^{2} z_{2}+R^{2} z_{1}\right)\left(z_{1}-z_{2}\right)},
\end{aligned}
$$

and, when we avoid the singularities, it turns into the equation

$$
\frac{\left(R^{2}-r_{1}^{2}\right)\left(R^{2}+r_{2}^{2}\right)^{2} m_{1}}{\left(R^{2}-r_{2}^{2}\right)\left(R^{2}+r_{1}^{2}\right)^{2} m_{2}}=-\frac{r_{2}^{2} z_{1}+R^{2} z_{2}}{r_{1}^{2} z_{2}+R^{2} z_{1}},
$$

which can be written as the polynomial equation

$$
\begin{aligned}
0 & =\left[m_{1}\left(R^{2}-r_{1}^{2}\right)\left(R^{2}+r_{2}^{2}\right)^{2} R^{2}+m_{2}\left(R^{2}-r_{2}^{2}\right)\left(R^{2}+r_{1}^{2}\right)^{2} r_{2}^{2}\right] z_{1} \\
& +\left[m_{1}\left(R^{2}-r_{1}^{2}\right)\left(R^{2}+r_{2}^{2}\right)^{2} r_{1}^{2}+m_{2}\left(R^{2}-r_{2}^{2}\right)\left(R^{2}+r_{1}^{2}\right)^{2} R^{2}\right] z_{2} .
\end{aligned}
$$


From (4.3) we can conclude that if one particle is fixed at the origin of the coordinate system, then the second particle moves along the geodesic circle with unitary velocity.

4.1. Two particles on the same circle. Since we have proved that for relative equilibria the bodies move along circles, we first assume that they are in the same circular orbit with radius $r_{1}=r_{2}=r$. We now have the following result.

Theorem 4.1. For the two-body problem in $\mathbb{M}_{R}^{2}$, a necessary and sufficient condition that the particles move along the same circle of radius $r \neq R$ and form $a$ relative equilibrium is that they have equal masses and are located at opposite sides of the circle.

Proof. Equation (4.3) becomes

$$
0=\left(R^{2}-r^{2}\right)\left(R^{2}+r^{2}\right)^{2}\left[\left(m_{1} R^{2}+m_{2} r^{2}\right) z_{1}+\left(m_{1} r^{2}+m_{2} R^{2}\right) z_{2}\right],
$$

which for $r \neq R$ is equivalent to

$$
0=\left(m_{1} R^{2}+m_{2} r^{2}\right) z_{1}+\left(m_{1} r^{2}+m_{2} R^{2}\right) z_{2} .
$$

If $m_{1}=m_{2}$, then from (4.5) we have

$$
0=m\left(R^{2}+r^{2}\right) z_{2}+m\left(r^{2}+R^{2}\right) z_{1},
$$

which implies that $z_{1}=-z_{2}$.

If in equation (4.5) we use the conditions $\left|z_{1}\right|=\left|z_{2}\right|=r$, we obtain

$$
r=\left|\frac{m_{1} R^{2}+m_{2} r^{2}}{m_{1} r^{2}+m_{2} R^{2}}\right| r,
$$

which implies that $m_{1}=m_{2}$, and therefore, again, that $z_{1}=-z_{2}$. It follows that the particles must be located at opposite sides of the circle of radius $r_{1}=r_{2}=r$.

Corollary 4.2. There are no relative equilibria for the two body problem such that one of the particles is located on the geodesic circle of radius $R$.

Proof. We observe that if one of the particles is on the geodesic circle, then from equation (4.3) we obtain that

$$
z_{2}=-\frac{r_{2}^{2}}{R^{2}} z_{1}
$$

which implies that $r_{2}=R$ and the particles must be antipodal, which is a forbidden configuration.

4.2. The general case. Here we study the more general problem of finding relative equilibria when the particles move on circles with different radii $r_{1} \neq r_{2}$ and will determine the relation between their masses. First we obtain the necessary and sufficient conditions in order to have nongeneric relative equilibria, which we call degenerate for short. The above degenerate relative equilibrium corresponds to a bifurcation for the family of relative equilibria of the two-body problem in $\mathbb{M}_{R}^{2}$.

Lemma 4.3. For the two-body problem in $\mathbb{M}_{R}^{2}$, a necessary and sufficient condition for the existence of a degenerate relative equilibrium is that the positions of the particles are given by

$$
z_{1}= \pm \sqrt{3 \pm 2 \sqrt{2}} R, \quad z_{2}= \pm \sqrt{3 \pm 2 \sqrt{2}} R
$$


and their mass relation is given by one of the equations

$$
m_{2}=m_{1}, \quad m_{2}=\frac{(2-\sqrt{2})^{2}}{(2+\sqrt{2})^{2}} m_{1}, \quad m_{2}=\frac{(2+\sqrt{2})^{2}}{(2-\sqrt{2})^{2}} m_{1} .
$$

Proof. To simplify the computations, but without loss of generality, we can fix the value of $m_{1}>0$ and take $z_{1}=r_{1}=\alpha$ to be determined later. Furthermore, if we take $\left|z_{2}\right|=r$ and $z_{2}=z$, then equation (4.3) becomes an algebraic equation in the variable $z$

$$
z=-\frac{m_{1}\left(R^{2}-\alpha^{2}\right)\left(R^{2}+r^{2}\right)^{2} R^{2}+m_{2}\left(R^{2}-r^{2}\right)\left(R^{2}+\alpha^{2}\right)^{2} r^{2}}{m_{1}\left(R^{2}-\alpha^{2}\right)\left(R^{2}+r^{2}\right)^{2} \alpha^{2}+m_{2}\left(R^{2}-r^{2}\right)\left(R^{2}+\alpha^{2}\right)^{2} R^{2}} \alpha .
$$

Notice that if $z_{1}=\alpha$ is located on the $x$-axis (it is a real number), then $z_{2}$ is also on the $x$-axis. Using the condition $|z|=r$ in equation (4.6), it follows that,

$$
0=\left[m_{1}\left(R^{2}-\alpha^{2}\right)\left(R^{2}+r^{2}\right)^{2} \alpha-m_{2}\left(R^{2}-r^{2}\right)\left(R^{2}+\alpha^{2}\right)^{2} r\right]\left(\alpha r \pm R^{2}\right) .
$$

Therefore

$$
r= \pm \frac{R^{2}}{\alpha}
$$

or

$$
m_{1} \alpha\left(R^{2}-\alpha^{2}\right)\left(R^{2}+r^{2}\right)^{2}-m_{2}\left(R^{2}-r^{2}\right)\left(R^{2}+\alpha^{2}\right)^{2} r=0 .
$$

In the first case, when we substitute relation (4.7) into equation (4.6), we obtain

$$
z_{2}=-\frac{R^{2}}{\alpha}
$$

which is a singular antipodal point with respect to $z_{1}=\alpha$ as defined in (3.2), and therefore cannot be a solution.

To search for real solutions of equation (4.8) and to prove the existence of relative equilibria, we define the real function of a real variable

$$
f(x)=m_{1} \alpha\left(R^{2}-\alpha^{2}\right)\left(R^{2}+x^{2}\right)^{2}-m_{2}\left(R^{2}+\alpha^{2}\right)^{2}\left(R^{2} x-x^{3}\right)
$$

and seek its zeros. For simplicity, we begin by studying the important case when the function $f$ has double roots. The derivative of that function is

$$
f^{\prime}(x)=4 m_{1} \alpha\left(R^{2}-\alpha^{2}\right)\left(R^{2}+x^{2}\right) x-m_{2}\left(R^{2}+\alpha^{2}\right)^{3}\left(R^{2}-3 x^{2}\right),
$$

and its common zeros with $f$ are the double roots of $f$. We obtain the double zeros by solving the system below in terms of $x$ :

$$
\begin{aligned}
m_{1} \alpha\left(R^{2}-\alpha^{2}\right)\left(R^{2}+x^{2}\right)^{2}-m_{2}\left(R^{2}+\alpha^{2}\right)^{2}\left(R^{2} x-x^{3}\right) & =0 \\
4 m_{1} \alpha\left(R^{2}-\alpha^{2}\right)\left(R^{2}+x^{2}\right) x-m_{2}\left(R^{2}+\alpha^{2}\right)^{3}\left(R^{2}-3 x^{2}\right) & =0 .
\end{aligned}
$$

Since $x=0$ is never a root of $f$ and $x^{2}+R^{2} \neq 0$, the above system is equivalent to

$$
\begin{aligned}
4 x f(x) & =0, \\
\left(R^{2}+x^{2}\right) f^{\prime}(x) & =0 .
\end{aligned}
$$

The solutions of this system are $x= \pm \sqrt{3 \pm 2 \sqrt{2}} R$. They are also conditions for having nontrivial solutions of the system (4.12) when viewed as a linear system in the variables $m_{1}$ and $m_{2}$. From the symmetry $\alpha \leftrightarrow x$ in equation (4.10), we also obtain the necessary condition for the value of $\alpha= \pm \sqrt{3 \pm 2 \sqrt{2}} R$. 
Therefore, in the particular case of double roots of $f$, the values

$$
z_{1}= \pm \sqrt{3 \pm 2 \sqrt{2}} R, \quad z_{2}= \pm \sqrt{3 \pm 2 \sqrt{2}} R
$$

belonging to the interval $(-\pi R, \pi R)$ give us the necessary conditions for the particles of masses $m_{1}$ and $m_{2}$ to form a relative equilibrium.

The mass relation is obtained from the first equation of the system (4.12),

$$
m_{2}=\frac{z_{1}\left(R^{2}-z_{1}^{2}\right)\left(R^{2}+z_{2}^{2}\right)^{2}}{z_{2}\left(R^{2}-z_{2}^{2}\right)\left(R^{2}+z_{1}^{2}\right)^{2}} m_{1}
$$

When we substitute the solutions (4.14) into (4.15) we obtain

$$
m_{2}=\frac{ \pm \sqrt{3 \pm 2 \sqrt{2}}(1 \pm \sqrt{2})(-1 \pm \sqrt{2})^{2}}{ \pm \sqrt{3 \pm 2 \sqrt{2}}(1 \pm \sqrt{2})(-1 \pm \sqrt{2})^{2}} m_{1} .
$$

Avoiding the singular points in (4.16) again, considering classes under the action of the group $\left\{H_{2}(t)\right\}$, and doing a simple (but tedious) analysis of the choice of signs, we obtain four possible different relative equilibria in $\mathbb{M}_{R}^{2}$ for this degenerated case with the mass relation

$$
m_{2}=m_{1}, \quad m_{2}=\frac{(2-\sqrt{2})^{2}}{(2+\sqrt{2})^{2}} m_{1}, \quad m_{2}=\frac{(2+\sqrt{2})^{2}}{(2-\sqrt{2})^{2}} m_{1} .
$$

For two equal masses there are two classes of relative equilibria: when the particles are inside the geodesic circle and when they are outside it. The last two relations in equation (4.17) correspond to one particle inside the geodesic circle and the other outside it. The last relation gives the bifurcation points of problem (3.4). This step completes the proof.

We can now state the main result of this section.

Theorem 4.4. Two particles with masses $m_{1}$ and $m_{2}$, located respectively at $z_{1}=a$ and $z_{2}=b$, and close enough to the circle of radius $\alpha$ in $\mathbb{M}_{R}^{2}$, form a relative equilibrium if and only if $a$ and $b$ are roots of (4.10), and the masses hold the relation

$$
m_{1} a\left(R^{2}-a^{2}\right)\left(R^{2}+b^{2}\right)^{2}=m_{2}\left(R^{2}-b^{2}\right)\left(R^{2}+a^{2}\right)^{2} b .
$$

Moreover, we can have $0,1,2,3$ or 4 classes of relative equilibria.

Proof. Using arguments of the theory of singularities and transversality (see Guillemin et al. 9]), we can perturb a little bit the degenerate case proved in Lemma 4.3, getting a sufficient condition to have new classes of relative equilibria. In this way, if we take a suitable perturbation in one of the masses (particularly the mass $m_{2}$ ), then the corresponding function $f$ in (4.10) can preserve one of the two tangential zeros, or generically, create two new roots in the interval $(-\pi R, \pi R)$ for each previous tangential zero. Each one of these new roots define a nondegenerate class of relative equilibrium. Now, if we take two of these new roots, say $z_{1}=a$ and $z_{2}=b$, then in order to have a relative equilibrium it is necessary to have the mass relation (4.18) obtained directly from (4.8). 


\section{THE CASE $n=3$}

For three particles interacting in $\mathbb{M}_{R}^{2}$, we obtain the system of algebraic equations which define the relative equilibria,

$$
\begin{aligned}
& \frac{R^{3}\left(R^{2}-r_{1}^{2}\right) z_{1}}{4\left(R^{2}+r_{1}^{2}\right)^{4}}=-\frac{m_{2}\left(r_{2}^{2}+R^{2}\right)^{2}\left(R^{2}+z_{1} \bar{z}_{2}\right)\left(z_{2}-z_{1}\right)}{\left\{\left(r_{1}^{2}+R^{2}\right)^{2}\left(r_{2}^{2}+R^{2}\right)^{2}-\left[2\left(z_{1} \bar{z}_{2}+z_{2} \bar{z}_{1}\right) R^{2}+\left(r_{1}^{2}-R^{2}\right)\left(r_{2}^{2}-R^{2}\right)\right]^{2}\right\}^{3 / 2}} \\
& -\quad \frac{m_{3}\left(r_{3}^{2}+R^{2}\right)^{2}\left(R^{2}+z_{1} \bar{z}_{3}\right)\left(z_{3}-z_{1}\right)}{\left\{\left(r_{1}^{2}+R^{2}\right)^{2}\left(r_{3}^{2}+R^{2}\right)^{2}-\left[2\left(z_{1} \bar{z}_{3}+z_{3} \bar{z}_{1}\right) R^{2}+\left(r_{1}^{2}-R^{2}\right)\left(r_{3}^{2}-R^{2}\right)\right]^{2}\right\}^{3 / 2}} \\
& \frac{R^{3}\left(R^{2}-r_{2}^{2}\right) z_{2}}{4\left(R^{2}+r_{2}^{2}\right)^{4}}=-\frac{m_{1}\left(r_{1}^{2}+R^{2}\right)^{2}\left(R^{2}+z_{2} \bar{z}_{1}\right)\left(z_{1}-z_{2}\right)}{\left\{\left(r_{1}^{2}+R^{2}\right)^{2}\left(r_{2}^{2}+R^{2}\right)^{2}-\left[2\left(z_{1} \bar{z}_{2}+z_{2} \bar{z}_{1}\right) R^{2}+\left(r_{1}^{2}-R^{2}\right)\left(r_{2}^{2}-R^{2}\right)\right]^{2}\right\}^{3 / 2}} \\
& -\frac{m_{3}\left(r_{3}^{2}+R^{2}\right)^{2}\left(R^{2}+z_{2} \bar{z}_{3}\right)\left(z_{3}-z_{2}\right)}{\left\{\left(r_{2}^{2}+R^{2}\right)^{2}\left(r_{3}^{2}+R^{2}\right)^{2}-\left[2\left(z_{2} \bar{z}_{3}+z_{3} \bar{z}_{2}\right) R^{2}+\left(r_{3}^{2}-R^{2}\right)\left(r_{2}^{2}-R^{2}\right)\right]^{2}\right\}^{3 / 2}} \\
& \frac{R^{3}\left(R^{2}-r_{3}^{2}\right) z_{3}}{4\left(R^{2}+r_{3}^{2}\right)^{4}}=-\frac{m_{1}\left(r_{1}^{2}+R^{2}\right)^{2}\left(R^{2}+z_{3} \bar{z}_{1}\right)\left(z_{1}-z_{3}\right)}{\left\{\left(r_{1}^{2}+R^{2}\right)^{2}\left(r_{3}^{2}+R^{2}\right)^{2}-\left[2\left(z_{1} \bar{z}_{3}+z_{3} \bar{z}_{1}\right) R^{2}+\left(r_{1}^{2}-R^{2}\right)\left(r_{3}^{2}-R^{2}\right)\right]^{2}\right\}^{3 / 2}} \\
& -\quad \frac{m_{2}\left(r_{2}^{2}+R^{2}\right)^{2}\left(R^{2}+z_{3} \bar{z}_{2}\right)\left(z_{2}-z_{3}\right)}{\left\{\left(r_{2}^{2}+R^{2}\right)^{2}\left(r_{3}^{2}+R^{2}\right)^{2}-\left[2\left(z_{2} \bar{z}_{3}+z_{3} \bar{z}_{2}\right) R^{2}+\left(r_{2}^{2}-R^{2}\right)\left(r_{3}^{2}-R^{2}\right)\right]^{2}\right\}^{3 / 2}} .
\end{aligned}
$$

5.1. Eulerian relative equilibria. First we study the collinear relative equilibria for the three-body problem. We consider the case when one particle of mass $m_{1}$ lies at the origin of coordinates $z_{1}=0$ and the other two particles with masses $m_{2}$ and $m_{3}$ are located at positions $z_{2}$ and $z_{3}$ on the same geodesic straight line. After a convenient rotation we can assume, without any loss of generality, that $z_{2}=a$ and $z_{3}=-r$, with $a, r$ positive constants. In this case, the equations of motions become

$$
\begin{aligned}
0 & =-\frac{m_{2}\left(a^{2}+R^{2}\right)^{2}}{a^{2}}+\frac{m_{3}\left(r^{2}+R^{2}\right)^{2}}{r^{2}}, \\
\frac{R^{3}\left(R^{2}-a^{2}\right) a}{4\left(R^{2}+a^{2}\right)^{4}} & =\frac{m_{1}}{8 R^{3} a^{2}}+\frac{m_{3}\left(r^{2}+R^{2}\right)^{2}\left(R^{2}-a r\right)(a+r)}{8 R^{3}(r+a)^{3}\left(a r-R^{2}\right)^{3}}, \\
\frac{R^{3}\left(R^{2}-r^{2}\right) r}{4\left(R^{2}+r^{2}\right)^{4}} & =-\frac{m_{1}}{8 R^{3} r^{2}}-\frac{m_{2}\left(a^{2}+R^{2}\right)^{2}\left(R^{2}-a r\right)(a+r)}{8 R^{3}(r+a)^{3}\left(a r-R^{2}\right)^{3}} .
\end{aligned}
$$

We start our analysis with the isosceles case, that is, we assume that $m=m_{2}=$ $m_{3}$ and $m_{1}=M$.

Theorem 5.1. A necessary and sufficient condition that a collinear configuration of the curved three-body problem in $\mathbb{M}_{R}^{2}$, with one particle at the origin, is a relative equilibrium is that the masses of the other two particles are equal and that the particles are located at opposite sides of the same circle.

Proof. From the first equation of system (5.2) we obtain

$$
\frac{\left(a^{2}+R^{2}\right)^{2}}{a^{2}}=\frac{\left(r^{2}+R^{2}\right)^{2}}{r^{2}},
$$

which implies that $r=a$ or $r=\frac{R^{2}}{a}$, and therefore $z_{3}=-a$ or $z_{3}=-\frac{R^{2}}{a}$. We avoid the last value because it is antipodal to $z_{2}=a$ and thus generates a singular point. Since $r=a$, both particles are on the same circle but at opposite sides.

If we assume that the particles with masses $m_{2}$ and $m_{3}$ are located on the same circle, then, using the fact that $a=\left|z_{3}\right|=r$, we obtain that necessarily 
$m_{2}=m_{3}=m$, which follows from the first equation of system (5.2). That is, both particles must have equal masses and must be located at opposite sides of the circle.

We recall that any straight line through the origin in $\mathbb{M}_{R}^{2}$ corresponds to a meridian geodesic circle in $\mathbb{S}_{R}^{2}$. We assume that the three particles are located on the same straight line and are rotating under the action of $G_{2}(t)$.

To obtain the condition for the existence of a relative equilibrium in terms of the masses, we substitute $r=a$ and $m_{1}=M$ in the second equation of (5.2) (or in the equivalent third one), and since $z_{2} \neq 0$, we obtain the relation for the radius $r$ in terms of the masses,

$$
\frac{R^{3} r\left(R^{2}-r^{2}\right)}{\left(R^{2}+r^{2}\right)^{4}}=\frac{M}{2 r^{2} R^{3}}-\frac{m\left(r^{2}+R^{2}\right)^{2}}{8 r^{2} R^{3}\left(r^{2}-R^{2}\right)^{2}} .
$$

This relation is equivalent with the polynomial equation

$$
4 M\left(R^{2}-r^{2}\right)^{2}\left(R^{2}+r^{2}\right)^{4}-m\left(r^{2}+R^{2}\right)^{6}-8 R^{6}\left(R^{2} r-r^{3}\right)^{3}=0
$$

with the mass relation

$$
M=\frac{\left(R^{2}+r^{2}\right)^{6} m+8 R^{6}\left(R^{2} r-r^{3}\right)^{3}}{4\left(R^{2}-r^{2}\right)^{2}\left(R^{2}+r^{2}\right)^{4}} .
$$

As in the case of the two-body problem in $\mathbb{S}_{R}^{2}$, the real solutions $r$ of equation (5.4) give us the circle where the particles with masses $m_{2}=m_{3}=m$ must move in order to form a relative equilibrium. The relation for these masses with the mass $m_{1}=M$ must satisfy equation (5.5).

We call this kind of orbit an isosceles Eulerian relative equilibria.

Now we will study the general collinear problem, that is, the case where the three masses are different. We have the following result.

Theorem 5.2. Consider a collinear configuration of the three-body problem in $\mathbb{M}_{R}^{2}$ with masses $m_{1}, m_{2}, m_{3}$, and suppose that the first particle with mass $m_{1}$ is located at $z_{1}=0$. Then there are two values $a^{*}$ and $a^{* *} \in[0, R]$ such that for every $a \in\left[0, a^{*}\right]$ and for any $a^{\prime} \in\left[a^{* *}, R\right]$ there exist two points $r$ and $r^{*}$ such that when we place the particles of masses $m_{2}, m_{3}$ at $z_{2}=a$ and $z_{3}=-r$ or at $z_{2}=a^{*}$ and $z_{3}=-r^{*}$ the corresponding configuration leads to a relative equilibrium if the masses satisfy the second or third equation of system (5.2).

Proof. From the first equation of system (5.2) we obtain

$$
\frac{m_{2}\left(a^{2}+R^{2}\right)^{2}}{a^{2}}=\frac{m_{3}\left(r^{2}+R^{2}\right)^{2}}{r^{2}},
$$

which is equivalent to

$$
\frac{a}{\sqrt{m_{2}}\left(a^{2}+R^{2}\right)}=\frac{r}{\sqrt{m_{3}}\left(r^{2}+R^{2}\right)} .
$$

We consider a couple of continuous real valued functions of the real variable:

$$
\begin{aligned}
& f_{2}(x)=\frac{1}{\sqrt{m_{2}}} \frac{x}{R^{2}+x^{2}}, \\
& f_{3}(x)=\frac{1}{\sqrt{m_{3}}} \frac{x}{R^{2}+x^{2}} .
\end{aligned}
$$


If $m_{3}<m_{2}$, then $f_{2}(x)<f_{3}(x)$ for all $x>0$. Both functions reach their maximum value at $x=R$; for the function $f_{2}(x)$ this corresponds to $f_{2}(R)=\frac{1}{2 \sqrt{m_{2}} R}$. Therefore for any $y \in\left(0, \frac{1}{2 \sqrt{m_{2}} R}\right)$ there are two points $r<R$ and $r^{*}>R$ such that equation (5.7) holds. For $a$ fixed, we solve equation (5.7) for $r$ in terms of $a$ obtaining

$$
\begin{aligned}
r & =\frac{\sqrt{\frac{m_{2}}{m_{3}}}\left(a^{2}+R^{2}\right)-\sqrt{\frac{m_{2}}{m_{3}}\left(a^{2}+R^{2}\right)^{2}-4 a^{2} R^{2}}}{2 a}, \\
r^{*} & =\frac{\sqrt{\frac{m_{2}}{m_{3}}}\left(a^{2}+R^{2}\right)+\sqrt{\frac{m_{2}}{m_{3}}\left(a^{2}+R^{2}\right)^{2}-4 a^{2} R^{2}}}{2 a},
\end{aligned}
$$

which are real numbers because $m_{3}<m_{2}$. Therefore the initial coordinates to obtain a collinear relative equilibrium are $\left(0, a, z_{3}\right)$, with $z_{3}=-r$ or $z_{3}=-r^{*}$ the values which solve equation (5.7). We observe that $z_{3}$ is not antipodal with $z_{2}=a$ because it is different from $-\frac{R^{2}}{a}$; therefore we have found a relative equilibrium with different masses. In the above reasoning we have fixed the value of $a$, but it is clear that not for all $z=a$ is it possible to obtain a relative equilibrium. Therefore, we must look for the necessary condition on $a$ in order to have a relative equilibrium, in this way multiplying both sides of the second equation of (5.2) by $m_{2}\left(a^{2}+R^{2}\right)^{2}$ and both sides of the third equation by $-m_{3}\left(r^{2}+R^{2}\right)^{2}$ imposing the relation (5.7). When we add the resultant equalities we get the relation

$$
m_{2} \frac{\left(R^{2}-a^{2}\right) a}{\left(R^{2}+a^{2}\right)^{2}}=m_{3} \frac{\left(R^{2}-r^{2}\right) r}{\left(R^{2}+r^{2}\right)^{2}} .
$$

As in the previous case we define the following auxiliary functions:

$$
\begin{aligned}
& g_{2}(x)=m_{2} \frac{\left(x^{2}-R^{2}\right) x}{\left(R^{2}+x^{2}\right)^{2}}, \\
& g_{3}(x)=m_{3} \frac{\left(x^{2}-R^{2}\right) x}{\left(R^{2}+x^{2}\right)^{2}},
\end{aligned}
$$

and we see that if $m_{3}<m_{2}$, then $g_{3}(x)<g_{2}(x)$ for all $x \in(0, R]$.

Let $\Lambda^{*}$ be the maximum of the function $g_{3}(x)$ in the interval $(0, R]$. Then, from the intermediate value theorem, there exist two points $a^{*}, a^{* *} \in(0, R]$ such that $g_{2}\left(a^{*}\right)=g_{2}\left(a^{* *}\right)=\Lambda^{*}$. This is because $g_{3}[0, R] \subset g_{2}[0, R]$ and both functions vanish at the ends of the interval. It follows that for any $a \in\left[0, a^{*}\right]$ there exists a unique point $r \in\left[0, a^{*}\right]$ such that

$$
m_{2} \frac{\left(a^{2}-R^{2}\right) a}{\left(R^{2}+a^{2}\right)^{2}}=g_{2}(a)=g_{3}(r)=m_{3} \frac{\left(r^{2}-R^{2}\right) r}{\left(R^{2}+r^{2}\right)^{2}},
$$

which implies that we have found a solution of (5.9). Using the same arguments we can show that for any $a^{*} \in\left[a^{* *}, R\right]$ there exists $r^{*} \in\left[a^{* *}, R\right]$ such that $g_{2}\left(a^{*}\right)=$ $g_{3}\left(r^{*}\right)$. Finally, we can obtain the relation for the masses using the second (or third) equation of (5.2).

5.2. Lagrangian solutions. In the classical Newtonian planar three-body problem it is well known that if three arbitrary masses are located at the vertices of an 
equilateral triangle and if suitable initial velocities are chosen, then this configuration generates a relative equilibrium called a Lagrangian solution. In the case of the three-body problem on the sphere $\mathbf{S}^{2}$ studied in [3], the authors proved that if three masses are located at the vertices of an equilateral triangle, then they generate a relative equilibrium only when the masses are equal. Moreover, they showed that the masses have to rotate on the same circle, whose plane must be orthogonal to the rotation axis. In [5] there is a complete analysis of the homographic solutions on the sphere, a class of solutions that include the relative equilibria.

Applying the stereographic projection and, since by Theorem 2.3 the equations of motion for the three-body problem on $\mathbf{S}^{2}$ and on $\mathbb{M}_{R}^{2}$ are equivalent for the analysis of the Lagrangian solutions on $\mathbb{M}_{R}^{2}$, it is enough to consider the case of three equal masses moving on the same circle of radius $r$. We will further show that this is in fact a necessary and sufficient condition for the existence of relative equilibrium.

Theorem 5.3. Let us consider a configuration of three equal masses moving on the same circle of radius $r$ in $\mathbb{M}_{R}^{2}$. Then a necessary and sufficient condition for the existence of a relative equilibrium is that the particles form an equilateral triangle configuration.

Proof. We consider three equal masses located on the same circle of radius $r$. Taking $z_{1}=r$ and $z_{2}, z_{3}$ on the same circle, the system of equations (5.1) becomes

$$
\begin{aligned}
\frac{R^{3}\left(R^{2}-r^{2}\right) r}{4 m\left(R^{2}+r^{2}\right)^{6}} & =-\frac{\left(R^{2}+r \bar{z}_{2}\right)\left(z_{2}-r\right)}{\left\{\left(r^{2}+R^{2}\right)^{4}-\left[2 r\left(\bar{z}_{2}+z_{2}\right) R^{2}+\left(r^{2}-R^{2}\right)^{2}\right]^{2}\right\}^{3 / 2}} \\
& -\frac{\left(R^{2}+r \bar{z}_{3}\right)\left(z_{3}-r\right)}{\left\{\left(r^{2}+R^{2}\right)^{4}-\left[2 r\left(\bar{z}_{3}+z_{3}\right) R^{2}+\left(r^{2}-R^{2}\right)^{2}\right]^{2}\right\}^{3 / 2}}, \\
\frac{R^{3}\left(R^{2}-r^{2}\right) z_{2}}{4 m\left(R^{2}+r^{2}\right)^{6}} & =-\frac{\left(R^{2}+r z_{2}\right)\left(r-z_{2}\right)}{\left\{\left(r^{2}+R^{2}\right)^{4}-\left[2 r\left(\bar{z}_{2}+z_{2}\right) R^{2}+\left(r^{2}-R^{2}\right)^{2}\right]^{2}\right\}^{3 / 2}} \\
& -\frac{\left(R^{2}+z_{2} \bar{z}_{3}\right)\left(z_{3}-z_{2}\right)}{\left\{\left(r^{2}+R^{2}\right)^{4}-\left[2\left(z_{2} \bar{z}_{3}+z_{3} \bar{z}_{2}\right) R^{2}+\left(r^{2}-R^{2}\right)^{2}\right]^{2}\right\}^{3 / 2}}, \\
\frac{R^{3}\left(R^{2}-r^{2}\right) z_{3}}{4 m\left(R^{2}+r^{2}\right)^{6}} & =-\frac{\left(R^{2}+z_{3} r\right)\left(r-z_{3}\right)}{\left\{\left(r^{2}+R^{2}\right)^{4}-\left[2 r\left(\bar{z}_{3}+z_{3}\right) R^{2}+\left(r^{2}-R^{2}\right)^{2}\right]^{2}\right\}^{3 / 2}} \\
& -\frac{\left(R^{2}+z_{3} \bar{z}_{2}\right)\left(z_{2}-z_{3}\right)}{\left\{\left(r^{2}+R^{2}\right)^{4}-\left[2\left(z_{2} \bar{z}_{3}+z_{3} \bar{z}_{2}\right) R^{2}+\left(r^{2}-R^{2}\right)^{2}\right]^{2}\right\}^{3 / 2}}
\end{aligned}
$$

Substitute $z_{2}=r e^{i \theta_{2}}$ and $z_{3}=r e^{i \theta_{3}}$ into equations (5.11), adding the first and third equation and subtracting from the sum the second equation twice. After separating the real and imaginary parts of the resulting equation we obtain the following system of equations:

$$
\begin{aligned}
\frac{1-\cos \theta_{2}}{D_{12}}+\frac{1-\cos \left(\theta_{3}-\theta_{2}\right)}{D_{23}} & =\frac{2\left(1-\cos \theta_{3}\right)}{D_{13}} \\
\frac{\sin \theta_{2}}{D_{12}}+\frac{\sin \left(\theta_{3}-\theta_{2}\right)}{D_{23}} & =0
\end{aligned}
$$


where

$$
\begin{aligned}
& D_{12}=8^{3 / 2} R^{3} r^{3}\left[1-\cos \theta_{2}\right]^{3 / 2}\left[R^{4}+r^{4}+2 r^{2} R^{2} \cos \theta_{2}\right]^{3 / 2}, \\
& D_{23}=8^{3 / 2} R^{3} r^{3}\left[1-\cos \left(\theta_{3}-\theta_{2}\right)\right]^{3 / 2}\left[R^{4}+r^{4}+2 r^{2} R^{2} \cos \left(\theta_{3}-\theta_{2}\right)\right]^{3 / 2}, \\
& D_{13}=8^{3 / 2} R^{3} r^{3}\left[1-\cos \theta_{3}\right]^{3 / 2}\left[R^{4}+r^{4}+2 r^{2} R^{2} \cos \theta_{3}\right]^{3 / 2} .
\end{aligned}
$$

If $\theta_{2}=\frac{2 \pi}{3}$ and $\theta_{3}=\frac{4 \pi}{3}$, then these values satisfy equations (5.12), and therefore the configuration corresponds to an equilateral triangle.

Using standard trigonometric identities in the first equation of (5.12), we obtain

$$
\left[\frac{\sin ^{2}\left(\frac{\theta_{3}-\theta_{2}}{2}\right)}{\sin ^{2}\left(\frac{\theta_{2}}{2}\right)}\right]^{2}\left[\frac{\left(r^{2}+R^{2}\right)^{4}-4 R^{2} r^{2} \sin ^{2}\left(\frac{\theta_{3}-\theta_{2}}{2}\right)}{\left(r^{2}+R^{2}\right)^{4}-4 R^{2} r^{2} \sin ^{2}\left(\frac{\theta_{2}}{2}\right)}\right]^{3}=\frac{1-\sin ^{2}\left(\frac{\theta_{3}-\theta_{2}}{2}\right)}{1-\sin ^{2}\left(\frac{\theta_{2}}{2}\right)} .
$$

Renaming the variables as $u=\sin ^{2}\left(\frac{\theta_{3}-\theta_{2}}{2}\right)$ and $v=\sin ^{2}\left(\frac{\theta_{2}}{2}\right)$, the above equation becomes

$$
u^{2}(1-v)\left(\left(r^{2}+R^{2}\right)^{4}-4 R^{2} r^{2} u\right)^{3}=v^{2}(1-u)\left(\left(r^{2}+R^{2}\right)^{4}-4 R^{2} r^{2} v\right)^{3} .
$$

This equation has only the real solution $u=v$, that is, $\sin ^{2}\left(\frac{\theta_{3}-\theta_{2}}{2}\right)=\sin ^{2}\left(\frac{\theta_{2}}{2}\right)$, or equivalently, $\theta_{3}=2 \theta_{2}$. If we substitute these values in the second equation of (5.12), we obtain

$$
\frac{1-\cos \theta_{2}}{1-\cos 2 \theta_{2}}=\frac{\left(R^{4}+r^{4}+2 r^{2} \cos 2 \theta_{2}\right)^{3}}{\left(R^{4}+r^{4}+2 r^{2} \cos \theta_{2}\right)^{3}} .
$$

Taking $w=1-\cos \theta_{2}$ and $s=1-\cos 2 \theta_{2}$, we are led to

$$
(1-w)\left(R^{4}+r^{4}+2 r^{2} w\right)^{3}=(1-s)\left(R^{4}+r^{4}+2 r^{2} s\right)^{3},
$$

which has only the real solution $w=s$, that is, $\cos \theta_{2}=\cos 2 \theta_{2}$, or equivalently

$$
\theta_{2}=0, \frac{2 \pi}{3}, \frac{4 \pi}{3}, 2 \pi
$$

Since we must avoid singular configurations, we have $\theta_{2}=\frac{2 \pi}{3}, \frac{4 \pi}{3}$, which correspond to an equilateral triangle.

\section{REFERENCES}

1. A.V. Borisov, I.S. Mamaev, A.A. Kilin, Two-body problem on a sphere: Reduction, stochasticity, periodic orbits, Regul. Chaotic Dyn. 9, 3 (2004), 265-279. MR2104172 (2005k:37199)

2. J. Cariñena, M. Rañada, M. Santander, Central potentials on spaces of constant curvature: The Kepler problem on the two dimensional sphere $\mathbb{S}^{2}$ and the hyperbolic plane $\mathbb{H}^{2}$, J. Math. Phys. 46 (2005), 1-17. MR2186791 (2007h:70020)

3. F. Diacu, E. Pérez-Chavela, M. Santoprete, The n-body problem in spaces of constant curvature, arXiv:0807.1747, (2008).

4. F. Diacu, On the singularities of the curved n-body problem. Trans. Amer. Math. Soc. 363, 4 (2011), 2249-2264. MR 2746682

5. F. Diacu, E. Pérez-Chavela, Homographic solutions of the curved 3-body problem. Journal of Differential Equations 250, 1 (2011), 340-366. MR2737846

6. F. Diacu, Polygonal Homographic Orbits of the Curved n-Body Problem. arXiv:1012.2490 (2010)

7. M. do Carmo, Differential Geometry of Curves and Surfaces, Prentice Hall, New Jersey, USA, 1976. MR 0394451 (52:15253)

8. B. Dubrovin, A. Fomenko, P. Novikov, Modern Geometry, Methods and Applications, Vol. I, II and III, Springer-Verlag, New York, 1984, 1985, 1990. MR0736837(85a:53003) MR0807945 (86m:53001) MR:1076994 (91j:55001)

9. V. Guillemin, M. Golubitsky, Stable mappings and their singularities, Springer-Verlag, New York, USA, 1973. MR0341518(49:6269) 
10. V.V. Kozlov, A.O. Harin, Kepler's problem in constant curvature spaces, Celestial Mech. Dynam. Astronom 54 (1992), 393-399. MR1188291 (93i:70010)

11. A.V. Shchepetilov, Comment on: Central potentials on spaces of constant curvature: The Kepler problem on the two-dimensional sphere $\mathbf{S}^{2}$ and the hyperbolic plane $\mathbf{H}^{2}$, J. Math. Phys. 46, 11 (2005), 052702. MR2186790(2007h:70019)

12. A.V. Shchepetilov, Nonintegrability of the two-body problem in constant curvature spaces, J. of Physics A: Mathematicas and general, 39, 5787-5806, 2006. MR2238116 (2007c:70014)

13. A.F. Stevenson, Note on the Kepler problem in a spherical space, and the factorization method for solving eigenvalue problems, Phys. Rev. 59 (1941), 842-843.

14. T.G. Vozmischeva, Intergrable problems of celestial mechanics in spaces of constant curvature, Kluwer Acad. Publ., Dordrecht, 2003. MR2027774 (2005a:37099)

Departamento de Matemáticas, UAm-Iztapalapa, Av. San Rafael Atlixco 186, México, D.F. 09340, MEXICO

E-mail address: epc@xanum.uam.mx

Departamento de Matemáticas, Uam-Iztapalapa, Av. San Rafael Atlixco 186, México, D.F. 09340, MExico

E-mail address: revg@xanum.uam.mx 